\title{
Structure and Properties of Fatigued Segmented Poly(urethaneurea)s I. Segment Orientation Mechanism due to Fatigue
}

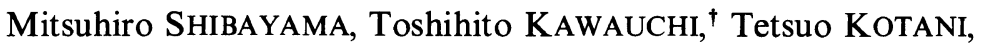 \\ Shunji NOMURA, and Takehisa MATSUDA* \\ Department of Polymer Science and Engineering, Faculty of Textile Science, \\ Kyoto Institute of Technology, Matsugasaki, Kyoto 606, Japan \\ * National Cardivascular Center Research Institute, \\ Suita, Osaka 565, Japan
}

(Received April 17, 1986)

\begin{abstract}
Changes of structure and properties of segmented poly(urethaneura)s (SPUU) subjected to a sinusoidal strain were studied as a function of the duration of the load (the fatigue time). Mechanical experiments as well as infrared dichroism experiments indicate that destruction of hard segment domains and phase mixing between hard and soft segment domains proceed with increasing fatigue time. Infrared dichroism shows that the hard segments orient negatively, then positively, with increasing elongation. Negative orientation results from the behavior of the hard segment domain as a filler having an anisotropic shape ("domain orientation"). Subsequent positive orientation is explained by the hard segment orientation coupled with fragmentation of the hard segment domains ("segment orientation"). In the fatigue process, on the other hand, proceeding of phase mixing is dominant followed by phase demixing related to rearrangement and reorientation of the hard segments, which would finally lead to fracture. It is found that the fatigue process consists of three stages; the domain orientation stage, the phase-mixing stage, and the segment orientation stage.
\end{abstract}

KEY WORDS Poly(urethaneurea) / Fatigue Mechanism / Infrared Dichroism / Spherulite Deformation /

Segmented poly(urethaneurea) (SPUU) block copolymers are thermoplastic copolymers which have mechanical properties similar to those of conventional crosslinked rubbers. These materials belong to a class of $(\mathrm{A}-\mathrm{B})_{n}$ type multiblock copolymers ${ }^{1-11}$ consisting of alternately joined blocks of two chemically dissimilar segments along the polymer backbone, which are called hard and soft segments.

Physical crosslinks are provided by the hard segments, which are generally formed from an extension of an aromatic diisocyanate with a low molecular weight diol or diamine. Elastomeric properties are impart- ed by the soft segments, usually formed from an aliphatic polyether or polyester macroglycol. Such SPUU is extensively hydrogen bonded. ${ }^{3-10}$ In all cases, the $\mathrm{NH}$ and $\mathrm{C}=\mathrm{O}$ groups of each urethane or urea linkage can act as a proton donor and a proton acceptor. Although hydrogen bonding is an important structural feature of these materials, its influence on their properties and morphologies is not clear. In addition, two-phase microdomain structure $^{7-10,12,13}$ has not been well understood due predominantly to the chemical complexity of the materials themselves. Characteristic mechanical properties of SPUU, such as, high extensibility, and resiliency,

\footnotetext{
† Present address: Sumitomo Rubber Industry Co., Ltd. Tsutsui-cho, Chuo-ku, Kobe 651, Japan.
} 
are attributed to the microphase separation of the hard and soft domains and hydrogen bonding related to urea and urethane groups.

Mechanical and thermal properties of SPUU were extensively studied by many workers. Wang et al. ${ }^{7}$ observed that mechanical properties depend on the content of the hard segment and an increase of the soft segment content leads to an isolated domain structure rather than an interconnected structure. It is often observed with DSC that an increase of the soft segment molecular weight lowers the domain mixing ("phase demixing"). Sung et al. ${ }^{1}$ and Wang et $a .^{7}$ found a three dimensional hydrogen bond which enhances aggregation of the hard segments.

Studies on hard and soft segment orientation are also extensively conducted in terms of infrared dichroism. Kimura et al..$^{5,14}$ and Cooper et al. ${ }^{10}$ observed that soft segments readily orient by an applied stress but hard segments have a tendency to orient negatively, and Kimura et al. proposed a spherulitic deformation model to account for this behavior.

Recently, SPUU has been focused on as medical material because of its compatibility with biomaterials and appropriate mechanical strength. These kinds of materials have been confirmed to have substantial compatibility ${ }^{13,15}$ with biomaterials, but the durability and the fatigue mechanism due to a cyclic deformation have not been understood. Therefore, the safety on durability has not been guaranteed.

The purpose of this series of papers is to investigate the morphological and orientational changes due either to an uniaxial elongation and to a repetitive sinusoidal strain, and to clarify the fatigue mechanism in SPUU. In this paper we study the variation of segment orientation and the degree of phase mixing in terms of mechanical experiments, differential scanning calorimetry (DSC) and infrared di- chroism.

\section{EXPERIMENTAL}

\section{Samples}

Two kinds of segmented poly(urethaneureas) (SPUU TM-1 and TM-3) (TOYOBO Co., Japan) consisting of poly(tetramethylene glycol) (PTMG), 4,4'-diphenylmethane diisocyanate (MDI), and 1,2-propylenediamine (PDA) are used for experiments. The chemical composition (in molar ratio) and the chemical structure of the SPUU's are presented in Table I. The number average molecular weights, $\bar{M}_{n}$, of PTMG are 856 and 1343, respectively for TM-1 and TM-3. Both TM-1 and TM-3 have the equal molar ratio of MDI, PTMG, and PDA. Dimethyl formamide (DMF) was used as a casting solvent.

As shown in Table I, TM-1 and TM-3 consist of soft and hard segments. The soft segments are formed by a sequence of PTMG and MDI, and are divided by urethane carbonyl groups at the junction of MDI and PTMG. The hard segments consist of MDI and PDA and have urea carbonyl groups.

Table I. Segmented poly(urethaneurea)s characterization

\begin{tabular}{cccc}
\hline Sample & $\begin{array}{c}\text { Soft segment } \\
\left(\bar{M}_{n}\right) \text { [PTMG] }\end{array}$ & MDI : PTMG:PDA & Solvent \\
\hline TM-1 & 856 & $2: 1: 1$ & DMF \\
TM-3 & 1343 & & \\
\hline
\end{tabular}

Soft segment (PTMG-MDI)

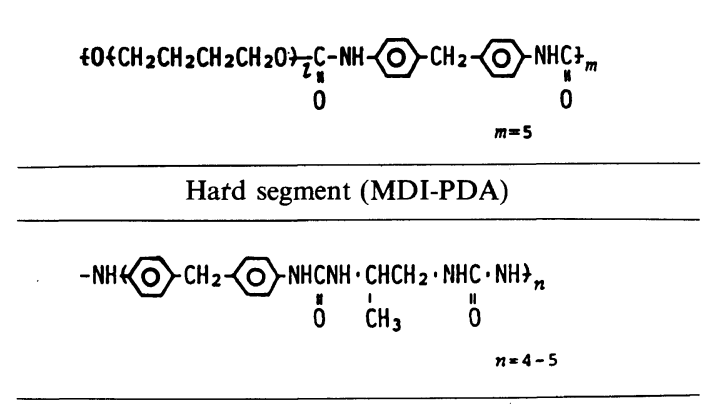


The average sequence numbers of the soft $(m)$ and hard segments $(n)$ were estimated to be about 5 and about 4 or 5 , respectively. ${ }^{5}$

Sample films are prepared as follows: A 2 or 10 weight-percent DMF solution was cast onto a clean glass plate followed by gradual solvent evaporation at $60^{\circ} \mathrm{C}$ in the atmosphere of the solvent vapor. A film on the glass was floated off on distilled water, put on a filter paper and then dried in a vacuum oven at $60^{\circ} \mathrm{C}$ for a week so as to completely remove residual solvent and moisture. The dried films were kept in a vacuum desiccator at room temperature until use. The thicknesses of the films were about $10 \mu \mathrm{m}$ and $300 \mu \mathrm{m}$ respectively according to the concentration of the initial solutions, i.e., $2 \%$ and $10 \%$. The thicknesses were measured by using the ratio between the object and the ocular micrometers under a microscope. The thick films of about $300 \mu \mathrm{m}$ were used only for the dynamic mechanical measurement, and the thin films of about $10 \mu \mathrm{m}$ thick were used for all other experiments due to strong infrared absorption of the film.

\section{Fatigue Test}

The as-cast samples of $50 \mathrm{~mm}$ long and $10 \mathrm{~mm}$ wide were subjected to a sinusoidal strain for a given time, i.e., the fatigue time. A fatigue testing apparatus was constructed for this purpose, which is a constant-strainamplitude type and has a counter indicating the number of cyclic straining. $100 \%$ of static strain and $20 \%$ of dynamic strain ${ }^{16}$ were employed for the fatigue test at $10 \mathrm{~Hz}$ and at $30^{\circ} \mathrm{C}$.

The fatigued samples as well as the as-cast sample were used for the mechanical, DSC, and infrared dichroism experiments. The fatigue time in thousand seconds is attached after the sample code, e.g., TM-1-10, indicating TM-1 fatigued for $10^{4}$ seconds $\left(10^{5}\right.$ load cycles).

\section{Mechanical Experiments}

Tensile tests were carried out with an Instron Model TOM200D (Shinko Tsushin Co., Ltd., Japan).

Dynamic mechanical measurements were conducted with a dynamic mechanical measurement apparatus. Data were collected at

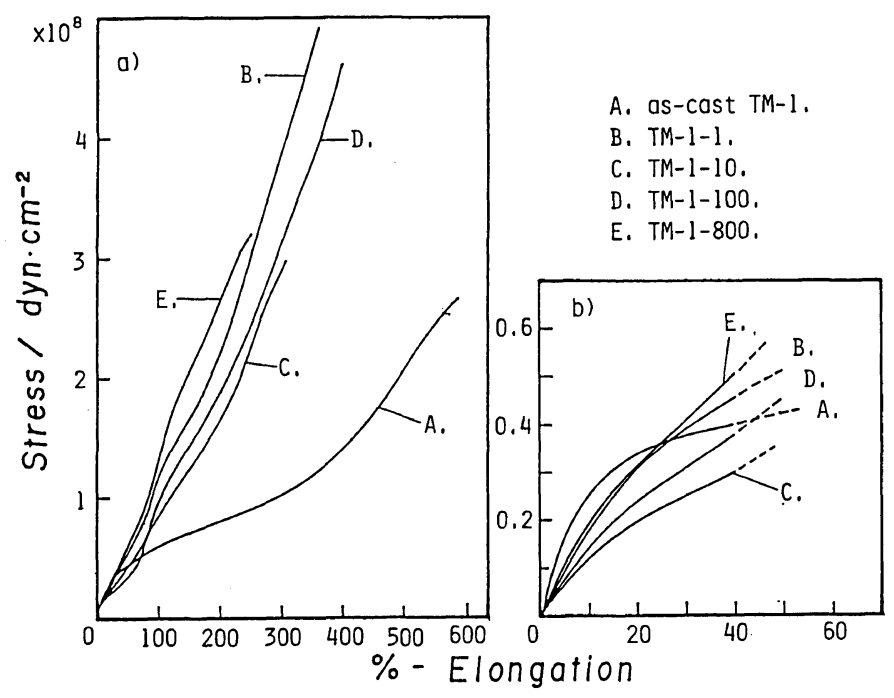

Figure 1. Stress-strain curves of the TM-1 series: (a, left) overall stress-strain curves until fracture; (b, right) initial portion of the stress-strain curves. 
nine frequencies over a wide frequency range between $2 \mathrm{~Hz}$ and $512 \mathrm{~Hz}$. Temperature was scanned with the heating rate of $2 \mathrm{~K} \mathrm{~min}^{-1}$ from $140 \mathrm{~K}$ to $350 \mathrm{~K}$. The strain imposed was in the order of a tenth percent.

\section{Differential Scanning Calorimetry (DSC)}

Differential scanning calorimetry was carried out with a DSC Model 8230 (Rigaku Co., Ltd.) at a heating rate of $10 \mathrm{~K} \mathrm{~min}^{-1}$ and a $\mathrm{N}_{2}$ purge of $50 \mathrm{ml} \mathrm{min} \mathrm{m}^{-1}$. About twenty pieces of the samples which weighed $50 \pm 5$ mg were crimped into an aluminum pan by a crimper. The DSC data were normalized with an equivalent sample weight.

\section{Infrared Studies}

Infrared absorption spectra were recorded with an infrared spectrophotometer DS-403G (Japan Spectroscopic Co., Ltd.). at a resolution of $10 \mathrm{~cm}^{-1}$. Infrared dichroism with stretching was performed with a stretching device and a silver chloride $(\mathrm{AgCl})$ polarizer inserted between the sample and the detector. The experiments were carried out at $20^{\circ} \mathrm{C}$ and at $65 \%$ relative humidity.

\section{RESULTS AND DISCUSSION}

\section{Mechanical Properties}

Figures $1 \mathrm{a}$ and $1 \mathrm{~b}$ show the stress-strain curves of four fatigued samples and of the ascast sample of TM-1, where Figure 1b shows the initial portion of the stress-strain curves. As shown in Figure 1a there is a clear difference between the as-cast sample (A) and the fatigued ones (B to E). It is interesting to see that the as-cast sample has lower stress-level and greater extensibility than the fatigued ones, and the initial modulus decreases and then increases with the fatigue time. These behavior is closely related to the changes in the internal structure which will be discussed later.

Similarly Figures $2 \mathrm{a}$ and $2 \mathrm{~b}$ show the stressstrain curves of TM-3. While the difference in the stress-strain behavior with respect to the fatigue time is not clear, it is noteworthy that Curve E shows the highest modulus and the lowest extensibility. Table II summarizes the tensile properties of TM-1 and TM3 series. Although the strength at break is scattered, the elongation at break clearly

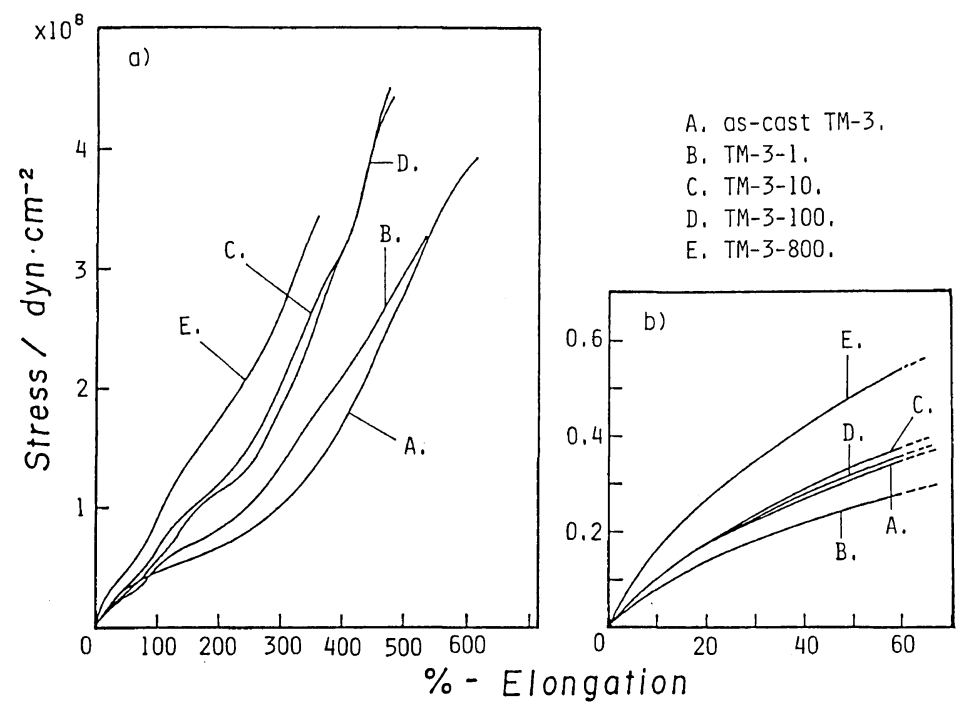

Figure 2. Stress-strain curves of the TM-3 series: (a, left) overall stress-strain curves until fracture; (b, right) initial portion of the stress-strain curves. 
Table II. The tensile properties of the TM-1 and TM-3 series

\begin{tabular}{|c|c|c|c|}
\hline \multirow{2}{*}{ Specimens } & $\begin{array}{l}\text { Strength at } \\
\text { break }\end{array}$ & $\begin{array}{l}\text { Elongation } \\
\text { at break }\end{array}$ & \multirow{2}{*}{$\begin{array}{l}\text { Modulus } \\
\text { dyn } \mathrm{cm}^{-2}\end{array}$} \\
\hline & dyn $\mathrm{cm}^{-2}$ & $\%$ & \\
\hline as-cast $\mathrm{T} \dot{\mathrm{M}}-1$ & $2.66 \times 10^{8}$ & 540 & $2.42 \times 10^{8}$ \\
\hline TM-1-1 & 4.92 & 360 & 2.36 \\
\hline TM-1-10 & 2.97 & 310 & 1.70 \\
\hline TM-1-100 & 4.69 & 410 & 2.32 \\
\hline TM-1-800 & 3.22 & 252 & 2.32 \\
\hline as-cast TM-3 & 3.95 & 636 & 1.11 \\
\hline TM-3-1 & 3.26 & 532 & 0.92 \\
\hline TM-3-10 & 4.52 & 480 & 1.16 \\
\hline TM-3-100 & 4.44 & 480 & 1.14 \\
\hline TM-3-800 & 3.46 & 360 & 1.88 \\
\hline
\end{tabular}

shows that the sample becomes brittle, the initial modulus decreases and then increases with increasing fatigue time for both TM-1 and TM-3 series. The difference in these tensile properties between TM-1 and TM-3 series is attributed to the difference in the hard segment content, supporting the similar results of Sung et al. ${ }^{1}$ and Wang et al. ${ }^{7}$

Tensile properties of elastomers have been extensively studied with a so-called Mooney-Rivlin plot. The Mooney-Rivlin equation gives the relationship between the stress $f$ and the extension ratio as follows,

$$
f /\left(\alpha-1 / \alpha^{2}\right)=2 C_{1}+2 C_{2} / \alpha
$$

where $\mathrm{C}_{1}$ and $C_{2}$ are constants. $C_{1}$ is known to be proportional to the cross-link density. The stress-strain behavior of TM-1 and TM-3 series is better characterized by the Mooney-Rivlin plot as shown in Figures 3a and $3 b$. Figure $3 a$ shows a drastic change of the mechanical properties of TM-1 with the fatigue time. The as-cast sample well follows the Mooney-Rivlin equation (eq 1) except at both high and low extension regions as shown with the dashed line. On the other hand, the fatigued samples showing up-turns around $1 / \alpha=0.6$ and/or 0.4 (as indicated with arrows) may follow eq 1 only at the low extension region. The up-turn behavior of

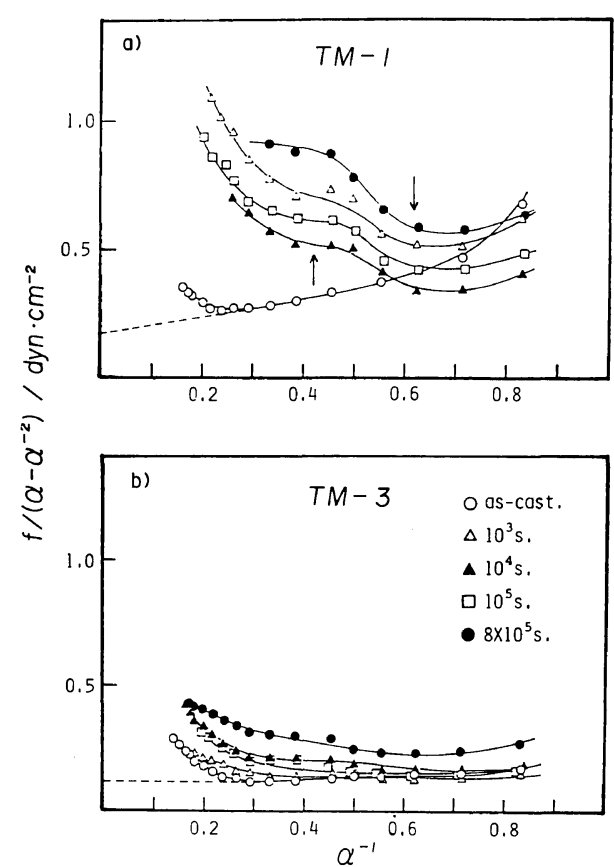

Figure 3. Mooney-Rivlin plots of the stress-strain curves: (a. top) the TM-1 series; (b. bottom) the TM-3 series.

$f /\left(\alpha-1 / \alpha^{2}\right)$ values with lowering $1 / \alpha$ may be due to fragmentation of lamellae, strainig and the orientational crystallization of the segments. The up-turn behavior at high $1 / \alpha$ (at low extension) region suggests the existence of an integrated structure of the hard segment domain particularly in the as-cast sample. These aspects will be discussed in Proposed Fatigue Mechanism. Figure 3b shows the Mooney-Rivlin plots of TM-3 series indicating that the $C_{1}$ value is lower, i.e., having lower crosslink density. Fatigue effect is less seen here.

Although the tensile properties of segmented poly(urethane ureas) greatly depends on the size, shape, and concentration of the hard domains as Cooper et al. ${ }^{7,17}$ pointed out, the tensile properties of the fatigued SPUU's studied here indicate complexity of the fatigue process.

The storage $\left(E^{\prime}\right)$ and the loss moduli $\left(E^{\prime \prime}\right)$ of TM-1 series at $2 \mathrm{~Hz}$ as a function of tem- 


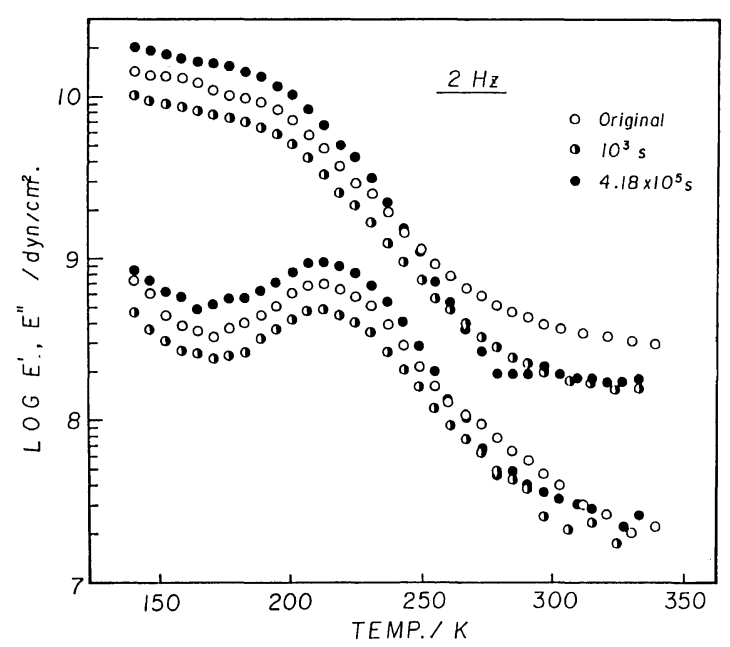

Figure 4. Temperature dependence of the storage modulus $\left(E^{\prime}\right)$ and the loss modulus $\left(E^{\prime \prime}\right)$ for fatigued TM-1 SPUU.

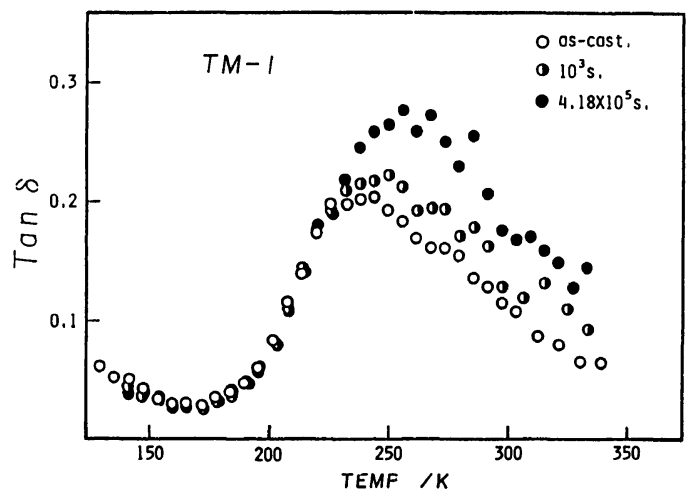

Figure 5. Reciprocal temperature dependence of the shift factor $\left(\log a_{\mathrm{T}}\right)$.

perature are shown in Figure 4. Each curve has similar behavior in this temperature range except its magnitude. The dispersion around $220 \mathrm{~K}$ corresponds to the glass transition of the soft segments, $T_{\mathrm{g}, \mathrm{s}}$, and that lower than $150 \mathrm{~K}$ might be related to the local mode motion of the PTMG. ${ }^{5,18,19}$ It is of interest that the storage $\left(E^{\prime}\right)$ and the loss moduli $\left(E^{\prime \prime}\right)$ decrease and then increase with increasing the fatigue time in the low temperature range. The difference in $E^{\prime}$ and/or $E^{\prime \prime}$ between the fatigued samples of $10^{3} \mathrm{~s}$ and $4.18 \times 10^{5} \mathrm{~s}$ comes up to twice as high. On the other hand, $E^{\prime}$ of the as-cast sample at the

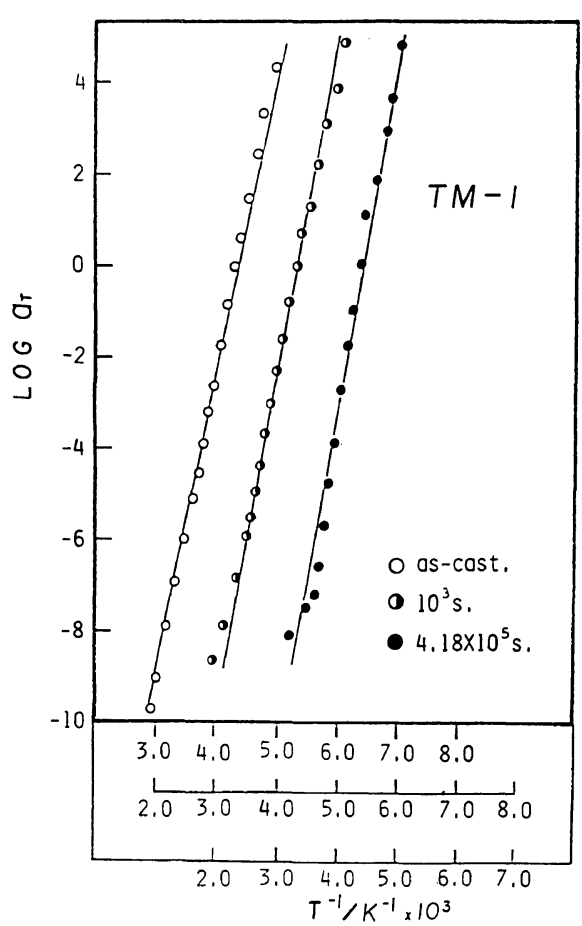

Figure 6. Temperature dependence of loss tangent, $\tan \delta$ of TM- 1 measured at $2 \mathrm{~Hz}$.

high temperature range has the highest value corresponding to the tensile properties at low extension (see Figure 1b). The dynamic mechanical properties also suggest a complicated structural change with fatigue. The fatigue process is well evaluated from the variation of the dissipation factor $(\tan \delta)$ as shown in Figure 5. As increasing the fatigue time the dispersion around $220 \mathrm{~K}$ increases and shifts toward higher temperature. This roughly corresponds to an increase of $T_{\mathrm{g}, \mathrm{s}}$ (see Table III).

The dynamic mechanical properties were studied over a wide frequency range $(2 \mathrm{~Hz}$ to $512 \mathrm{~Hz}$ ) and a temperature range $(140 \mathrm{~K}$ to $350 \mathrm{~K}$ ) as mentioned in EXPERIMENTAL, and a master curve was constructed. Figure 6 shows the reciprocal temperature dependence of the shift factor, $\log a_{\mathrm{T}}$ obtained from the superposition of the frequency dispersions of $E^{\prime}$ and $E^{\prime \prime}$. As can be seen in the figure, $\log a_{\mathrm{T}}$ for each sample has a linear 
relationship with the reciprocal temperature, suggesting an existence of a single dispersion process in the temperature range between $200 \mathrm{~K}\left(1 / T=0.005 \mathrm{~K}^{-1}\right)$ and $330 \mathrm{~K} \quad(1 / T=$ $\left.0.003 \mathrm{~K}^{-1}\right)$. The activation energies were $34.6,36.5$, and $33.4 \mathrm{kcal} \mathrm{mol}^{-1}$ for the as-cast $\mathrm{TM}-1, \mathrm{TM}-1-10^{3}$, and TM-1-4.18 $\times 10^{5}$, respectively.

\section{Thermal Analysis}

Figures 7 and 8 show the DSC thermograms of TM-1 series and TM-3 series, respective-

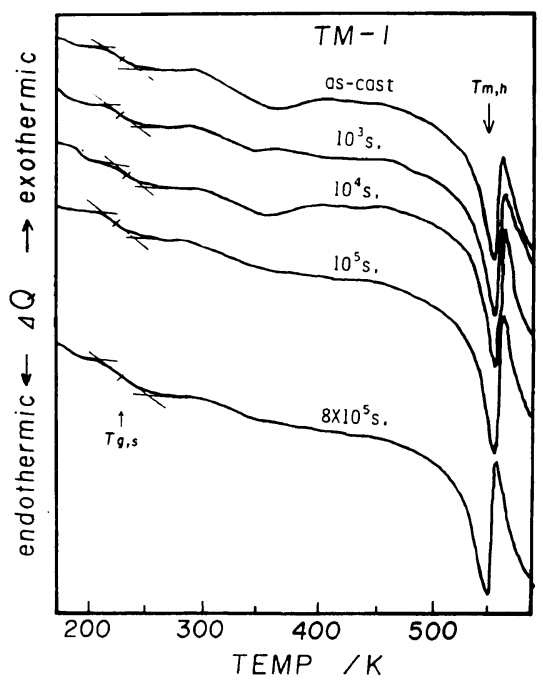

Figure 7. DSC curve of TM-1 series. ly. There are several characteristic temperatures, $5,7,11,20$ such as the glass transition temperature of the soft segments, and the hard segment melting temperature, $T_{\mathrm{m}, \mathrm{h}}$, as indicated in the figures. In addition to these, the soft segment melting endotherm $T_{\mathrm{m}, \mathrm{s}}$ is seen in TM-3 series. The endotherm beginning from around $560 \mathrm{~K}$ is due to degradation of the samples. These characteristic temperatures as well as the heat of fusion of the hard segments, $\Delta Q_{\mathrm{h}}$, are listed in Table III. Clear difference is seen between TM-1 and TM-3

Table III. Thermal characteristics of TM-1 and ГM-3 series obtained with DSC

\begin{tabular}{|c|c|c|c|c|}
\hline \multirow{2}{*}{ Specimens } & \multirow{2}{*}{$\frac{T_{\mathrm{g}, \mathrm{s}}}{\mathrm{K}}$} & \multirow{2}{*}{$\frac{T_{\mathrm{m}, \mathrm{s}}}{\mathrm{K}}$} & \multirow{2}{*}{$\frac{T_{\mathrm{m}, \mathrm{h}}}{\mathrm{K}}$} & \multirow{2}{*}{$\frac{\text { Heat of fusion, } \Delta Q_{\mathrm{h}}}{\mathrm{mj} \mathrm{mg}^{-1}}$} \\
\hline & & & & \\
\hline as-cast TM-1 & 224.0 & - & 547.5 & 17.39 \\
\hline TM-1-1 & 225.8 & - & 547.0 & 21.49 \\
\hline TM-1-10 & 227.4 & - & 548.5 & 22.06 \\
\hline TM-1-100 & 225.5 & - & 548.5 & 18.87 \\
\hline TM-1-800 & 228.3 & - & 540.0 & 26.66 \\
\hline as-cast TM-3 & 209.5 & 267.9 & 545.6 & 13.27 \\
\hline TM-3-1 & 209.0 & 266.2 & 551.0 & 14.25 \\
\hline TM-3-10 & 209.8 & 269.0 & 552.0 & 12.12 \\
\hline TM-3-100 & 209.0 & 267.4 & 535.6 & 8.81 \\
\hline TM-3-800 & 210.0 & 267.0 & 549.5 & 14.15 \\
\hline
\end{tabular}

$T_{\mathrm{m}, \mathrm{s}}:$ Soft segment melting point.

$T_{\mathrm{m}, \mathrm{h}}$ : Hard segment melting point. 


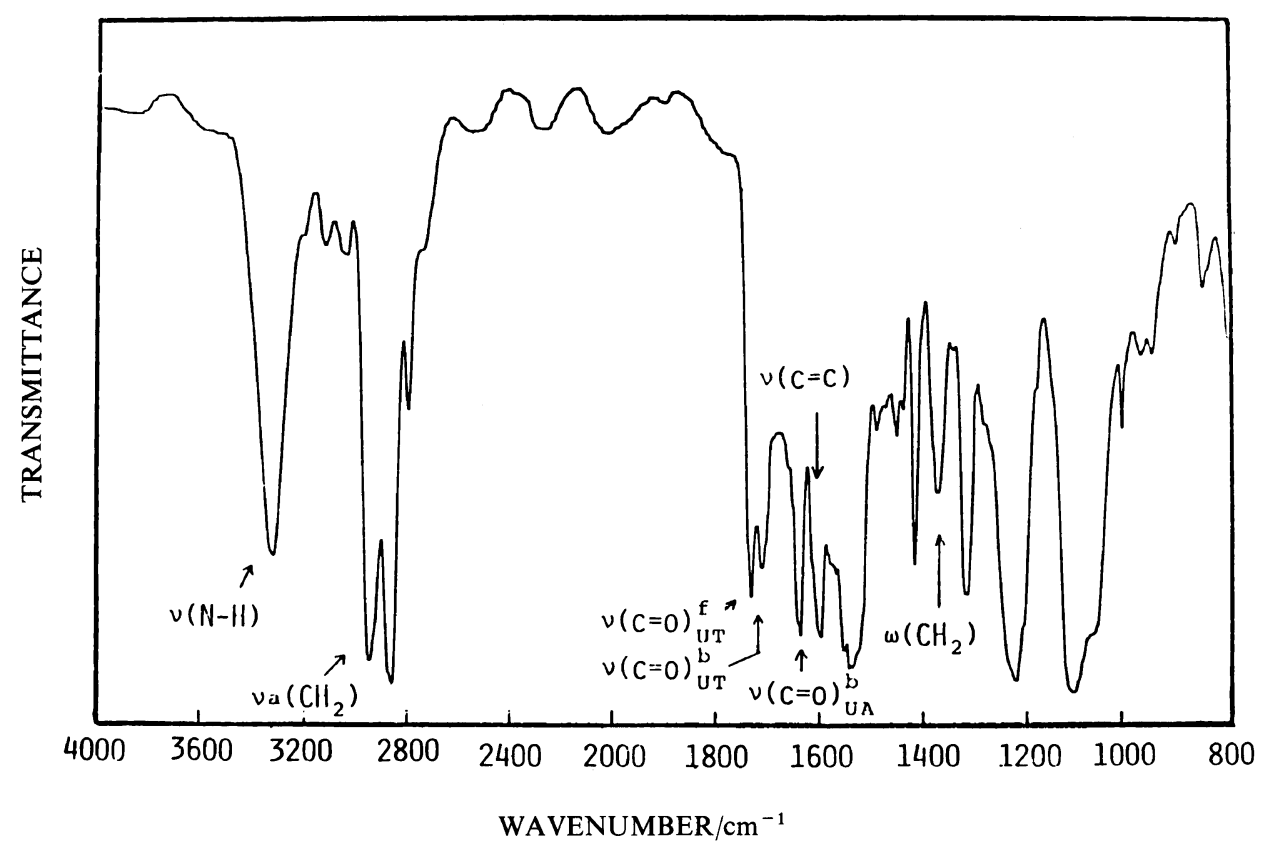

Figure 9. Infrared (IR) survey spectrum of the as-cast TM-1.

series. The glass transition temperatures of TM-1 series are higher than those of TM-3. There exist the melting points of the soft segments in TM- 3 series. The melting points of the hard segments, $T_{\mathrm{m}, \mathrm{h}}$, of TM-3 series are higher than those of TM-1 series. All of these suggest that the phase separation between the hard and soft segments is more developed in TM-3 series than in TM-1 series. This is simply due to the longer soft segments of TM3 series. The segment length effects on the structure were well studied by Wang et al. ${ }^{7}$ and Sung et al. ${ }^{20}$

Although the changes of these thermal characteristics with increase of the fatigue time are better seen in TM-1 series than in TM-3 series, neither of them clearly indicates a systematic change of the structure. This may be due to complicated structural changes with fatigue such as, orientation changes of the hard and soft segments, association or dissociation of hydrogen bonds and to the difficulty of DSC measurement of very thin films.

\section{Infrared Studies}

Hydrogen Bond Indices. Figure 9 shows the infrared (IR) survey spectrum of the ascast TM-1. Similar spectra were obtained from the as-cast TM-3 and all of the fatigued samples. Characteristic bands are indicated with symbols. The $\mathrm{NH}$ absorption peak $v(\mathrm{~N}-\mathrm{H})$ located at $3320 \mathrm{~cm}^{-1}$ indicates that most of the $\mathrm{NH}$ groups are hydrogen bonded because the free $\mathrm{NH}$ absorption peak at $3445 \mathrm{~cm}^{-1}$ is negligible. In the carbonyl region between 1620 and $1750 \mathrm{~cm}^{-1}$, the absorption peak at $1730 \mathrm{~cm}^{-1}, v(\mathrm{C}=\mathrm{O})_{\mathrm{UT}}^{\mathrm{f}}$, is due to the free urethane carbonyl groups, and that of $1710 \mathrm{~cm}^{-1}, v(\mathrm{C}=\mathrm{O})_{\mathrm{UT}}^{\mathrm{b}}$, is due to the hydrogen bonded urethane carbonyl groups. The absorption peak located at 1640 $\mathrm{cm}^{-1}, v(\mathrm{C}=\mathrm{O})_{\mathrm{UA}}^{\mathrm{b}}$, is corresponding to the hydrogen bonded urea carbonyl groups. The absorption peak due to the free urea carbonyl groups at $1695 \mathrm{~cm}^{-1}$ was not observed. Therefore the urea carbonyl groups are considered to be completely hydrogen bonded, which is also supported by the $\mathrm{NH}$ absorption 
behavior.

The relative absorbance of the bonded and the free carbonyl groups gives the fraction of the hydrogen bond in urethane groups $H_{\mathrm{UT}}$, which is defined as,

$$
H_{\mathrm{UT}}=\frac{A(1710)}{A(1710)+A(1730)}
$$

where $A(1710)$ and $A(1730)$ are the average absorbances of the bonded and the free carbonyl absorption peaks at 1710 and 1730 $\mathrm{cm}^{-1}$, respectively. ${ }^{5,7,12}$

On the other hand, the ratio of the relative absorbance of the hydrogen bonded urea carbonyl absorption peak to that of the $\mathrm{C}=\mathrm{C}$ stretching absorption peak of the benzene ring at $1600 \mathrm{~cm}^{-1}$ is used as an index of the degree of the hydrogen bond of the urea carbonyl groups in the hard segments. ${ }^{5,13} \mathrm{In}$ another word, that ratio could be used as an index of the degree of the cohesive force of the hard segments. The cohesive force index of the hard segments, $C_{\mathrm{h}}$, is given by ${ }^{12,19}$

$$
C_{\mathrm{h}}=\frac{A(1640)}{A(1600)}
$$

where $A(1640)$ and $A(1600)$ are the average absorbances at $1640 \mathrm{~cm}^{-1}$ and at $1600 \mathrm{~cm}^{-1}$, respectively. The variation of $H_{\mathrm{UT}}$ and $C_{\mathrm{h}}$ will be discussed as a function of the fatigue time and the elongation ratio.

Infrared Dichroism. The asymmetric $\mathrm{CH}_{2}$ stretching band at $2940 \mathrm{~cm}^{-1}$ and the $\mathrm{C}=\mathrm{O}$ stretching band at $1640 \mathrm{~cm}^{-1}$ were used to study the orientation of the soft and the hard segments, respectively. The transition moment vectors of asymmetric vibration of $\mathrm{CH}_{2}$ and $\mathrm{C}=\mathrm{O}$ stretching bands are reported to be oriented by about $90^{\circ}$ and $78^{\circ}$, respectively, with respect to the segment (or the chain) axes. ${ }^{21} \mathrm{We}$ studied, however, the orientation behavior of these bands instead of those of the soft and hard segments. Then the dichroic orientation factor $F_{i}^{\mathrm{D}}$ for the $i$-th band is simply obtained with the following equation,

$$
F_{i}^{\mathrm{D}}=\frac{1-D_{i}}{1+2 D_{i}}
$$

where $D_{i}$ is the dichroic ratio

$$
D_{i}=\frac{A_{i, \perp}}{A_{i, \|}}
$$

$A_{i, \|}$ and $A_{i, \perp}$ are the absorbances measured with radiations polarized parallel and perpendicular to the stretching direction, respectively. The positive $F_{i}^{\mathrm{D}}$ means the negative orientation of the corresponding segments and vice versa due to the relationship between the corresponding segments axes and the transition moments for the perpendicular dichroism. For the simplicity in data manipulation, straight base lines were assumed at range of $3680-2520 \mathrm{~cm}^{-1}$ for the asymmetric $\mathrm{CH}_{2}$ band and of $1950-900 \mathrm{~cm}^{-1}$ for

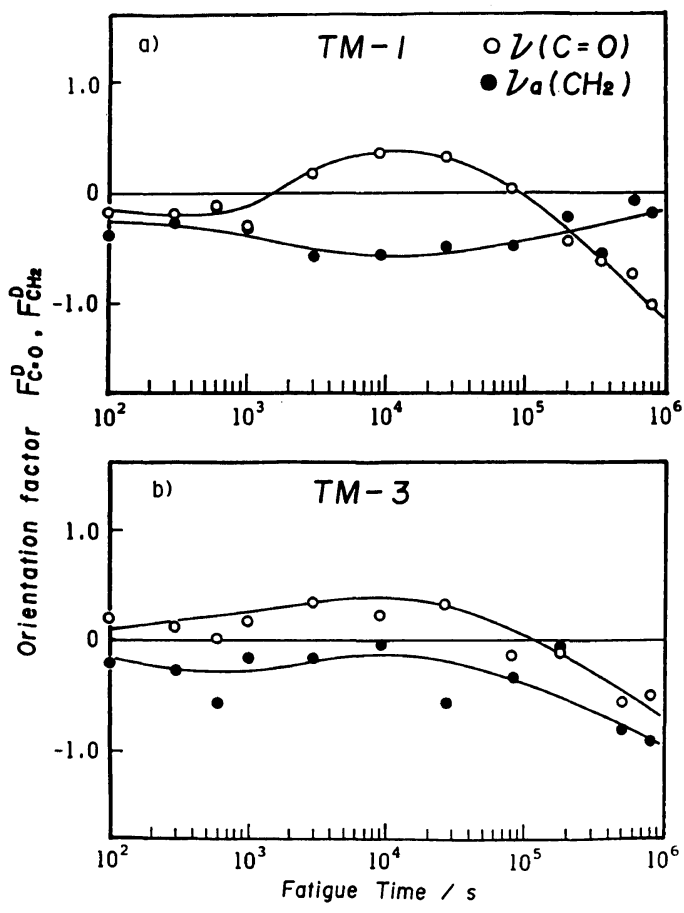

Figure 10. Fatigue time dependence of the orientation factor, $F_{\mathrm{C}=\mathrm{O}}^{\mathrm{D}}$ and $F_{\mathrm{CH}_{2}}^{\mathrm{D}}$ in terms of the $v(\mathrm{C}=\mathrm{O})$ and $v_{\mathrm{a}}$ $\left(\mathrm{CH}_{2}\right)$, respectively for the TM-1 series (a, top) and for the TM-3 series (b, bottom). 
the $\mathrm{C}=\mathrm{O}$ stretching band.

Fatigue Time Dependence of the Orientation Behavior and of the Hydrogen Bond Indices, $H_{\mathrm{UT}}$ and $C_{\mathrm{h}}$. Figures $10 \mathrm{a}$ and $10 \mathrm{~b}$ show the fatigue time dependence of the orientation behavior of the hard and soft segments in terms of the orientation factors $F_{\mathbf{C H}_{2}}^{\mathrm{D}}$ and $F_{\mathrm{C}=\mathrm{O}}^{\mathrm{D}}$. The sample is relaxed after load. The difference in the orientation factors between TM-1 and TM-3 is again clear (see Figure 3, for example). In the case of TM-1, the hard segments having the urea $\mathrm{C}=\mathrm{O}$ group orient positively up to the fatigue time of $10^{3} \mathrm{~s}$, change to the negative orientation having a maximum at $10^{4} \mathrm{~s}$, and then orient positively again with increasing the fatigue time. This negative orientation of the hard segments is of interest. Similar behavior is found in TM-3 except the earlier fatigue time
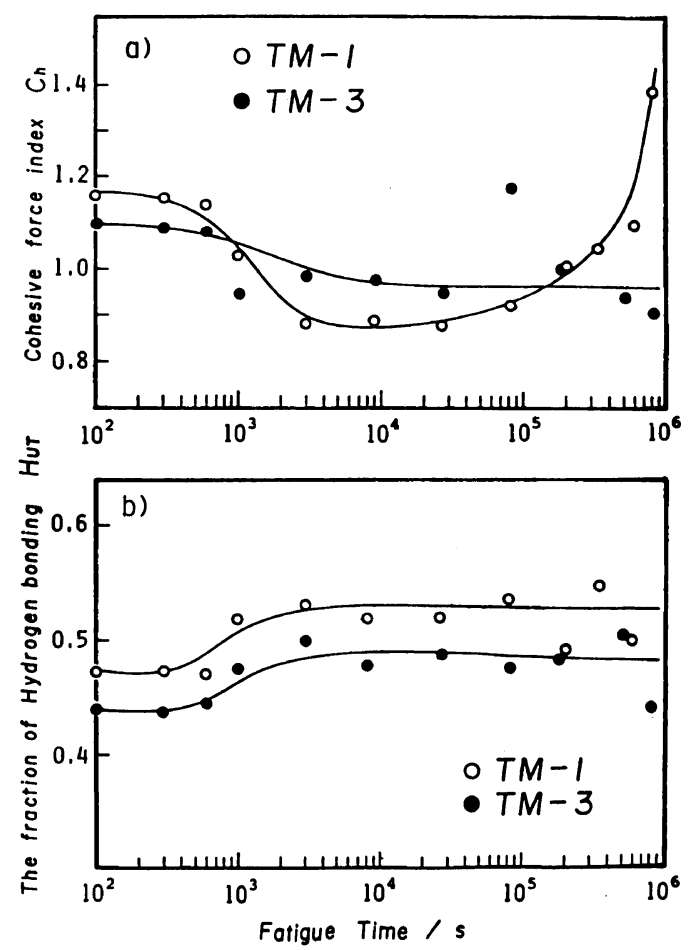

Figure 11. Fatigue time dependence of the cohesive force index, $C_{\mathrm{h}}(\mathrm{a}$, top) and the fraction of the hydrogen bonding of urethane carbonyls (b, bottom). region. On the other hand, the soft segments keep positive orientation over the whole range of the fatigue time and the variation of the orientation factors is less compared with those of the hard segments for both TM-1 and TM-3. This suggests that the hard segments are much affected by the fatigue, in this case, by the sinusoidal strain. TM-1 was broken at about $1.5 \times 10^{6} \mathrm{~s}$ when it was further subjected to the load. Therefore the strong positive orientation of the hard segment at longer fatigue time than $10^{6}$ would lead a fracture of the system probably due to an overstraining of the soft segments when the sample is stretched (see Figure 13). The opposite behavior of the soft segment orientations between TM- 1 and TM-3 at the fatigue time longer than $10^{6} \mathrm{~s}$ is not accounted for at this stage.

The structural change in the hard segments at the late stage of the fatigue process is clearly seen also in the change of the cohesive force index $C_{\mathrm{h}}$ in Figure 11a. The cohesive force index of TM-1 shows the dissociation of the hard segments which causes the phase mixing at the fatigue time range of $10^{3} \mathrm{~s}$ to $10^{5} \mathrm{~s}$. The abrupt change in $C_{\mathrm{h}}$ at the fatigue time longer than $10^{5}$ suggests the hard segments aggregate and are rearranged due to the load in which the orientation of the hard segments play a lot of roles. The change of the Young's modulus with the fatigue time is corresponding to the chages of the orientation of the hard segments and the cohesive force index. The change of the cohesive force index of TM-3 is less than that of TM-1, which may be due to the fact that the longer soft segments in TM-3 lower the internal strain, showing no up-turn of $C_{\mathrm{h}}$ in this time range.

Figure $11 \mathrm{~b}$ shows the change of the fraction of the hydrogen bond in the urethane group $H_{\mathrm{UT}}$. $H_{\mathrm{UT}}$ of TM-1 is higher than that of TM-3, which means the urethane carbonyl groups of TM-1 are much hydrogen bonded in numbers than those of TM-3. In addition, the phase mixing in the soft segment 


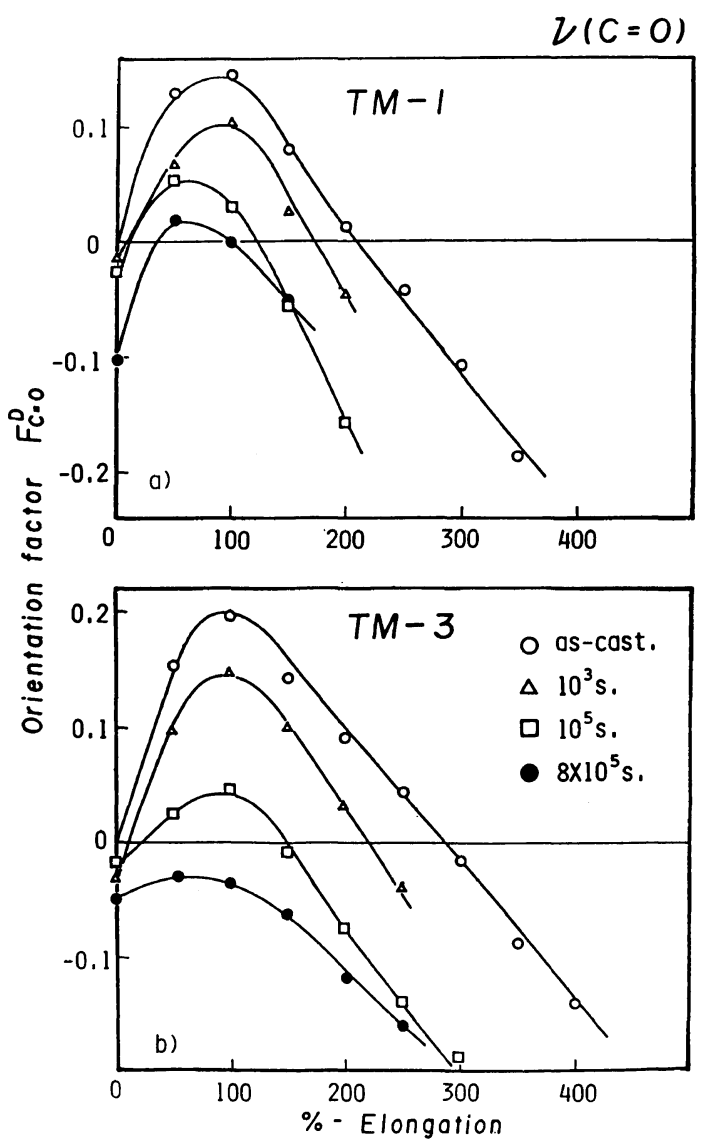

Figure 12. $\%$-Elongation dependence of the orientation factor in respect to the $(\mathrm{C}=\mathrm{O})$ : $(\mathrm{a}, \mathrm{top})$ for the TM-1 series; (b, bottom) for the TM-3 series.

domain is more developed in the similar manner both for TM-1 and TM-3 with the fatigue time. One may conclude that the change of the degree of phase mixing with fatigue in the soft segment domain is different from that in the hard segment domain, i.e., an asymmetric phase mixing occurs.

Elongation Ratio Dependence of the Orientation Behavior and the Hydrogen Bond Indices. Figures $12 \mathrm{a}$ and $12 \mathrm{~b}$ respectively show the orientation factor $F_{\mathrm{C}=\mathrm{O}}^{\mathrm{D}}$ of the urea carbonyl groups for TM-1 and TM-3 as a function of elongation. Both of the as-cast TM-1 and TM-3 show the negative orientation at low elongation. The maxima of the negative orientation lie around $100 \%$ elongation. Then

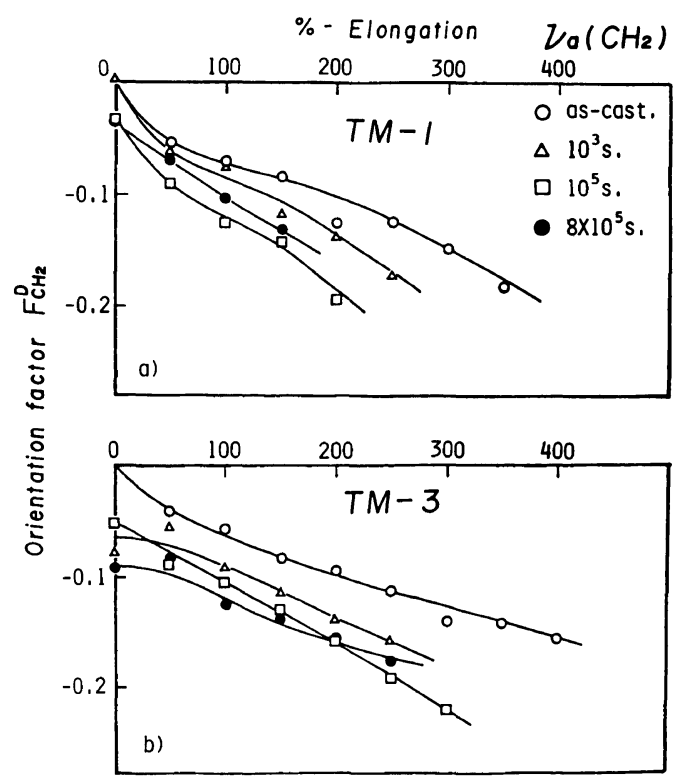

Figure 13. $\%$-Elongation dependence of the orientation factor in respect to the $\left(\mathrm{CH}_{2}\right)$ : (a, top) for the TM1 series; (b, bottom) for the TM-3 series.

the hard segments orient positively with further elongation. This negative orientation behavior of the hard segments is always observed in uniaxial stretching of segmented polyurethane derivatives. ${ }^{5,7,14}$ The degree of the negative orientation differs with changing of the lengths of the hard and soft segments ${ }^{7,14}$ as well as the chemical structure of the segments..$^{5,7}$

With increasing fatigue time, a negative offset of the orientation factor $F_{\mathrm{C}=\mathrm{O}}^{\mathrm{D}}$ appears at zero-elongation, which means the hard segments orient positively with increasing fatigue. Once stretched, however, the hard segments show the negative orientation similarly to the case of the as-cast samples. The maximum of $F_{\mathrm{C}=\mathrm{O}}^{\mathrm{D}}$ shifts toward lower elongation with increasing the fatigue time.

Figures $13 \mathrm{a}$ and $13 \mathrm{~b}$ show the elongation dependence of the orientation factor in respect to the band related to asymmetric stretching of $\mathrm{CH}_{2}$. The soft segments orient positively with increasing elongation. The trend of fatigue time dependence of the soft segment 
orientation is negligible for both TM-1 and TM-3 except positive off-sets at zero-elongation.

The combination of the negative orientation of the hard segments and the positive orientation of the soft segments at low elongation is widely observed. ${ }^{5,7,14,22}$ Two mechanisms to account for this behavior have been proposed. One is the Bonart's model ${ }^{22,23}$ in which the hard segment lamellae having the principal axis in the direction perpendicular to the hard segment orient parallel to the stretching direction resulting in the negative orientation of the hard segments. The other is the spherulite deformation model proposed by Kimura et al ${ }^{5,14}$ Bonart's model is based on assumptions that the lateral size of the hard segment lamellae is much bigger than the thickness of the lamellae and the continuum mechanical moment is bigger than the chain mechanical moment. This rotation mechanism has

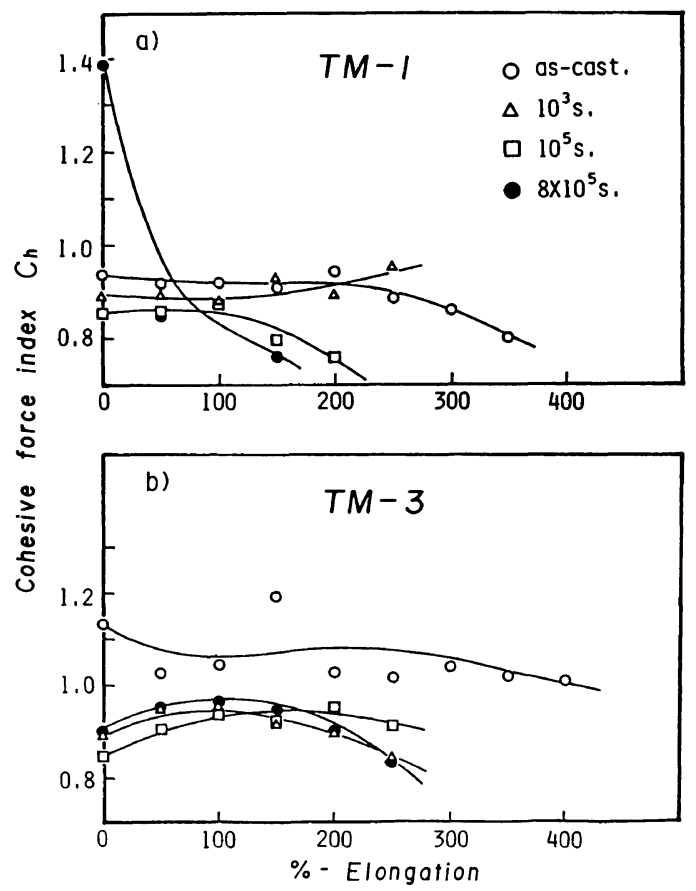

Figure 14. $\%$-Elongation dependence of the cohesive force idex, $C_{\mathrm{h}}$ of the hard segments: (a, top) TM-1 series; (b, bottom) TM-3 series. not been proven with experimental evidences. The spherulite deformation model is based on the spherulite deformation of crystalline polymers which forms spherulitic textures. ${ }^{5,14,24}$ The latter model is more plausible if the system has spherulitic textures. The deformation of the spherulites can be observed in terms of light scattering technique or other techniques as studied by Kimura et al. ${ }^{5,14}$ In the case of the segmented poly(urethaneureas) having low content of the hard segments, the existence of the spherulitic textures are uncertain. Such systems, however, also exhibit the negative orientation of the hard segments. Therefore the exact mechanism of the deformation behavior of segmented poly(urethaneureas) does not seem to be clarified.

In the case of TM-1 and TM-3, spherulitic structures were found in the thick film with small angle light scattering technique, ${ }^{25}$ but not in the thin film. This may be simply due to

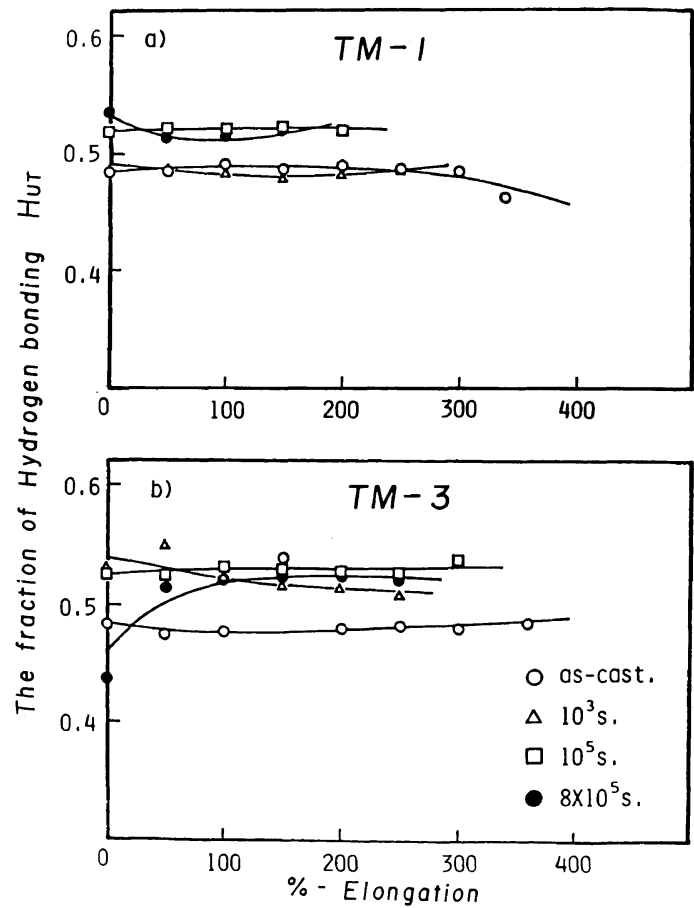

Figure 15. $\%$-Elongation dependence of the fraction of the hydrogen bonding, $H_{\mathrm{UT}}$ of urethane carbonyls: (a, top) TM-1 series; (b, bottom) TM-3 series. 
the low optical contrast between the spherulites and the matrix. If one still accepts the spherulite deformation mechanism, the fatigue process means the destruction of the spherulitic textures, which is corresponding to the change of the cohesive force index $C_{\mathrm{h}}$ and the lowering of the negative orientation with the fatigue time and to the phase mixing developed with the fatigue time.

Figures 14a and 14b show the cohesive force indices for TM-1 and TM-3, respectively, as a function of elongation. The cohesive force index seems to have little elongation and fatigue time dependence except TM-1-800. Similar behavior is found in the elongation dependence of the fraction of the hydrogen bonding $H_{\mathrm{UT}}$ as shown in Figures $15 \mathrm{a}$ and $15 \mathrm{~b}$.

\section{Proposed Fatigue Mechanism}

Figure 16 shows the fatigue time dependence of the Young's modulus, the orientation factor $F_{\mathrm{C}=\mathrm{O}}^{\mathrm{D}}$, and the cohesive force index $C_{\mathrm{h}}$ for TM-1. This figure suggests that the

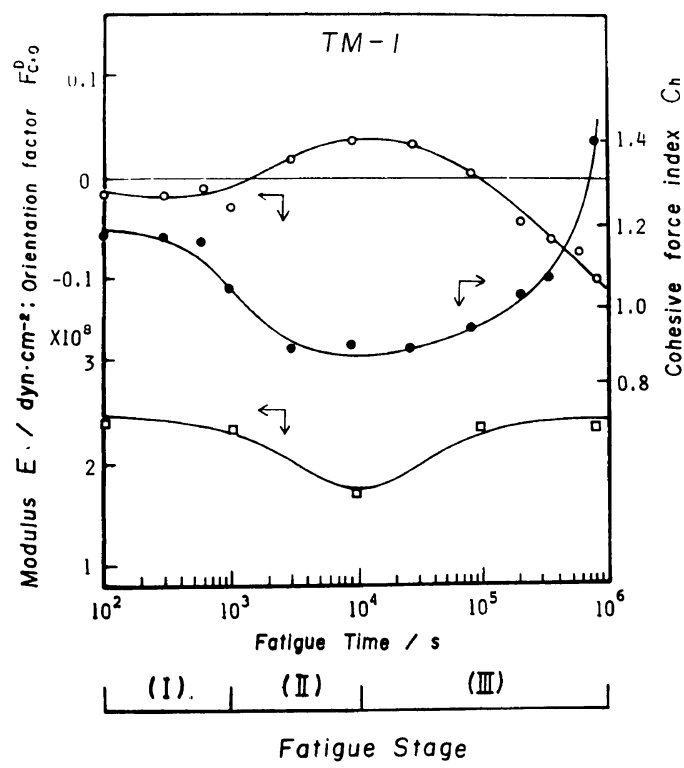

Figure 16. Fatigue time dependence of the Young's modulus, the orientation factor $F_{\mathrm{C}=\mathrm{O}}^{\mathrm{D}}$, and the cohesive force index $C_{\mathrm{h}}$. The fatigue stages are indicated at the bottom. fatigue process can be divided into three stages as indicated at the bottom of the figure. In Stage I, the system keeps more or less the original structure. In Stage II, the decrease of $C_{\mathrm{h}}$ indicates the proceeding of phase mixing. The orientation factor $F_{\mathrm{C}=0}^{\mathrm{D}}$, however, indicates the existence of spherulites (in the spherulite deformation model) or the lamellae which can orient along stretching direction (in Bonart's model). In Bonart's model lamellae orient along the stretching direction where the hard segments orient negatively with respect to the stretching direction and then the lamellae are broken up to small domains or fragments. Further stretching leads positive orientation of the hard segments. Therefore the positive orientation of the hard segments should occur at the same time with the lowering of the cohesive force index. Experimental results show the positive orientation begins in Stage III whereas the lowering of $C_{\mathrm{h}}$ occurs in Stage II, which is not accounted for by the Bonart's model and requires the existence a higher texture than the lamellae structure. If one accepts the spherulite deformation model, the fatigue process is interpreted as follows: First stretch breaks a part of the spherulite, e.g., the equatorial region. The structure, however, is more or less preserved in Stage I. In Stage II the region where the hard segment domains are broken up to smaller domains becomes large resulting in the lowering of the cohesive force index. On the other hand, the other parts of the spherulites still keeping the spherulitic textures follow the spherulitic deformation. This is a kind of "domain orientation" proposed by Hashimoto et al. ${ }^{25}$ in the case of deformation of block copolymers. In Stage III, the spherulites are completely broken except at the polar region and then rearranged and reaggregate to a fringed-micell-like structure parallel to the stretching direction ("segment orientation") resulting in increases of the modulus and the cohesive force index. All of these are supported by the small angle light 
and X-ray scattering analyses which will be reported in the forthcoming papers. ${ }^{25}$

\section{CONCLUDING REMARKS}

The fatigue mechanism as well as the deformation mechanism was studied with mechanical, DSC, and IR dichroism experiments. The stress-strain curves of TM-1 show a distinct fatigue effect. The fatigue makes the TM-1 film brittle. The long soft segments lowers this effect. Dynamic mechanical measurements indicate that the storage and loss moduli decrease and then increase with the fatigue time, suggesting the existence of a three step fatigue process at least. DSC curves show complicated changes in the characteristic parameters, which need further justification. IR dichroism was found to be one of the most powerful techniques to investigate the fatigue process of segmented poly(urethaneureas) because IR dichroism provides independent information on the hard and soft domain orientations and on the state of aggregation.

The orientation behavior and the cohesive force index in the urea group changed markedly in TM-1 series with the fatigue time, suggesting TM-1 having a higher hard segment content is easily affected by the fatigue. It was found that the hard segment is selectively fatigued with a sinusoidal strain. The negative orientation behavior of the hard segments is explained with the spherulite deformation model better than with the Bonart's model. It is found that the fatigue process of TM-1 consists of three stages; the initial lowfatigued stage (Stage I), the phase mixing stage (Stage II), and the fatigue-induced micellation stage (Stage III). In Stage I the structure is more or less preserved. In Stage II the phase mixing proceeds, and the hard segments show the positive orientation (domain orientation). In Stage III the rearrangement of the hard segment domains occurs due to the orientation change of the segments along the stretching direction (segment orientation) resulting in aggregation of the hard segments. This process is well interpreted with the employment of the spherulite deformation model.

Acknowledgment. We express our sincere thanks to Toyobo Katata Research Institute, Otsu, Shiga, Japan, for kind supply of the samples. We aknowledge partial financial support from the Ministry of Education, Science and Culture, Japan, Under Grants 60750825.

\section{REFERENCES AND NOTE}

1. C. S. Paik-Sung, T. W. Smith, and H. H. Sung, Macromolecules, 13, 117 (1983).

2. C. M. Brunette, S. L. Hsu, and W. J. MacKnight, Macromolecules, 15, 71 (1982).

3. G. M. Estes, R. W. Seymour, and S. L. Cooper, Macromolecules, 4, 452 (1971).

4. J. L. Blackwell and K. H. Gardner, Polymer, 20, 13 (1979).

5. I. Kimura and H. Ishihara, "Kagakuzoukan," No. 58, Kagakudojin, Kyoto, 1973, p 208; H. Ono and I. Kimura, ibid., No. 50, p 181.

6. J. L. Blackwell, M. R. Nagarajan, and T. B. Hoitink, Polymer, 23, 950 (1982).

7. C. B. Wang and S. L. Cooper, Macromolecules, 16, 775 (1983).

8. K. Matsumoto, I. Kimura, K. Saito, and H. Ono, Rep. Prog. Polym. Phys. Jpn., 13, 205 (1970).

9. A. L. Chang and E. L. Thomas, J. Polym. Sci., 8, 33 (1979).

10. S. L. Cooper and R. W. Seymour, "Block and Graft Copolymers," J. J. Burke and V. Weiss, Ed., Syracuse Univ. Press, New York, N.Y., 1973.

11. C. S. Schollenberg, J. Polym. Sci., 8, 83 (1979).

12. A. Takahara, J. Tashita, T. Kajiyama, and M. Takayanagi, Polym. Prepr., Jpn., 32, No. 8 (1983).

13. I. D. Fridman and E. L. Thomas, Polymer, 21, 388 (1980).

14. I. Kimura, H. Ishihara, H. Ono, N. Yoshihara, S. Nomura, and H. Kawai, Macromolecules, 7, 355 (1974).

15. T. Matsuda, T. Akutsu, and S. Nomura, Polym. Prepr., Jpn., 31, No. 8 (1983).

16. It is clear that the fatigue mechanism is dependent on the magnitudes of static and dynamic strains as well as the frequency and temperature. The experimental condition in this study was chosen in order to observe the fatigue process within the time scale of observation, particularly around $100 \%$ elongation where an interesting structural change occurs as 
shown in Figure 12. Instantaneous structural change occurs at the first stretch followed by gradual changes due to an repetitive stimulus.

17. J. A. Koutsky, N. V. Hein, and S. L. Cooper, J. Polym. Sci., B, 8, 353 (1970).

18. T. Matsuda, H. Iwata, and T. Akutsu, Polym. Prepr., Jpn., 32, No. 8 (1983).

19. A. Takahara, J. Tashita, T. Kajiyama, and M. Takayanagi, Kobunshi Ronbunshu, 39, 203 (1982).

20. C. S. Sung, D. B. Hu, and C. S. Wu, Macromolecules, 13, 111 (1980).
21. G. M. Estes, R. W. Seymour, and S. L. Cooper, Macromolecules, 4, 452 (1975).

22. F. Hoffman and R. Bonart, Makromol. Chem., 184, 1529 (1983).

23. R. Bonart, J. Macromol. Sci. Phys., 132, 115 (1968).

24. S. Nomura, H. Kawai, I. Kimura, and M. Kajiyama, J. Polym. Sci., A-2, 5, 479 (1967).

25. M. Shibayama, Y. Ohki, and S. Nomura, to be submitted.

26. T. Pakula, K. Saijo, H. Kawai, and T. Hashimoto, Macromolecules, 8, 1294 (1985). 